\title{
Long Run Risks in the Term Structure of Interest Rates : Estimation
}

\author{
Taeyoung Doh \\ Federal Reserve Bank of Kansas City *
}

February 5, 2008

\begin{abstract}
This paper estimates a long run risk model with term structure data. Inflation and consumption growth both contain correlated long run risk components. The model is estimated by the likelihood-based Bayesian methods and estimates of the latent long run risk factors are extracted from both macro and term structure data. Empirical analysis using US data reveals that a small and persistent component in consumption growth interacting with expected inflation improves the model's fit for the term structure data.

JEL CLASSIFICATION: C32, E43, G12

KEY WORDS: $\quad$ Long Run Risks Bayesian Econometrics

Term Structure of Interest Rates
\end{abstract}

*Taeyoung Doh : Research Department, Federal Reserve Bank of Kansas City, 925 Grand Blvd, Kansas City, MO 64198; email: Taeyoung.Doh@kc.frb.org. Brent Bundick provided excellent research assistance regarding construction of data. The views expressed herein are solely those of the author and do not necessarily reflect the views of the Federal Reserve Bank of Kansas City or the Federal Reserve System. 


\section{Introduction}

Long run risk models along the line of Bansal and Yaron (2004) can account for asset pricing puzzles such as equity premium and low risk-free rate. A small but persistent component in expected consumption growth combined with fluctuating economic uncertainty and Epstein-Zin (1989) type recursive preferences shed new light on economic sources of risks priced in financial markets. Recently, Piazzesi and Schneider (2006) and Bansal and Shaliastovich (2007) apply a similar framework to explain bond markets and show some progress in matching term structure moments. However, neither study estimates a long run risk model by using macro and term structure data at the same time. Bansal and Shaliastovich (2007) calibrate parameters governing shock processes and preference while Piazzesi and Schneider (2006) estimate parameters of shock processes from only macro data and calibrate preference parameters to match term structure moments. In this paper, I estimate a long run risk model with both macro and term structure data and provide estimates of the long run risk components by filtering out short run factors. One advantage of this approach is that it provides clear evidence of the long run risk factors implied by the model.

In the empirical analysis using US data, I find that a small but persistent part in consumption growth together with the real impacts of inflation and recursive preferences can improve the model's fit. The model implied pricing errors are smaller than values reported in a no-arbitrage affine term structure model using macro factors (e.g. Bikbov and Chernov (2006)). In particular, the real impacts of inflation seem to be important in explaining term structure data in contrast to Bansal and Shaliastovich (2007) who shut down this channel. Smoothed estimates of long run consumption risk seem to be less volatile but more persistent than observed consumption growth. This result is consistent with the key assumption of long run risk models. On the other hand, long run risk component in inflation is as volatile as inflation itself and the model implies a bit more volatile inflation process. As I discuss below, this result is not confined to the long run risk structure of the model but more general to various classes of macro-finance models of term structure. 
The paper is closely related to recent literature on the estimation of long run risk models. Bansal, Gallant, and Tauchen (2007) estimate a long run risk model of Bansal and Yaron (2004) and an external habit model of Campbell and Cochrane (1999) and compare the empirical plausibility of two models. Using aggregate stock market return, they show that the two models fit data much better than a standard power utility version but the long run risk model better reflects the co-movements between consumption and returns. Bansal, Kiku, and Yaron (2007) estimate preference parameters of a long run risk model from data on the cross-section of stock market returns and find that low frequency movements and time-varying volatility in consumption growth can explain the returns of various portfolios and their pricedividend ratios. However, none of these previous works explores term structure implications of long run risk models. And direct estimates of long run components are not given in Bansal, Gallant, and Tauchen (2007) while Bansal, Kiku, and Yaron (2007) extract a long run risk component in consumption from asset market data by regressing observed consumption growth on the previous period's observed market return. It is not clear how accurately the procedure would recover long run risk components if we consider the existence of pricing errors in asset market data. Indeed, when I use regression-based estimates of long run risk components, the implied persistence is lower and the predictability of consumption growth is higher than the case when long run risk factors are backed out as latent factors through filtering. In a closely related paper, Chen, Favilukis, and Ludvigson (2007) estimate an asset pricing model based on recursive preferences. Unlike long run risk models, they do not impose tight parametric restrictions on the law of motion or distribution of consumption. Even in this more generalized version, they find fluctuations in expected consumption growth provides a better description of asset market data, which is consistent with the basic message of long run risk models.

I proceed as follows: Section 2 describes the model economy and derives the approximate solution of equilibrium bond yields. Section 3 presents the econometric methodology used in the estimation. Section 4 provides estimation results based on empirical analysis of US data. Section 5 contains concluding remarks. Details of the derivation of equilibrium bond yields and econometric methodology are given in 
a technical appendix.

\section{Model}

\subsection{Preference and Shocks}

Following Bansal and Yaron (2004), I consider a representative-agent with EpsteinZin (1989) recursive preferences in an endowment economy with complete marktes. More specifically, the representative agent's preference over the consumption stream is given by:

$$
U_{t}=\left[(1-\delta) C_{t}^{\frac{1-\gamma}{\theta}}+\delta\left(E_{t} U_{t+1}^{1-\gamma}\right)^{\frac{1}{\theta}}\right]^{\frac{\theta}{1-\gamma}}
$$

The time discount factor $(\delta)$, the risk-aversion $(\gamma \geq 0)$, and the intertemporal elasticity of substitution (IES $: \psi \geq 0$ ) characterize the preference with $\theta$ as $\frac{1-\gamma}{1-\frac{1}{\psi}}$. The standard expected utility is a special case of the above recursive preferences when $\gamma$ is equal to $\frac{1}{\psi}$.

Epstein-Zin (1989) shows that the logarithm of the nominal stochastic discount factor in the recursive preferences has the following form:

$$
m_{t+1}=\theta \log \delta-\frac{\theta}{\psi} g_{c, t+1}+(\theta-1) r_{c, t+1}-\pi_{t+1}
$$

Here, $g_{c, t+1}$ is the log growth rate of aggregate consumption and $r_{c, t+1}$ is the log of the return on an asset which pays aggregate consumption as its dividends. $\pi_{t+1}$ is the inflation rate at $t+1$. $r_{c, t+1}$ is not directly observable but can be approximated as a function of state variables governing the dynamics of $g_{c, t+1}$ and $\pi_{t+1}$ by using the following no-arbitrage restriction.

$$
E_{t}\left(e^{m_{t+1}+\pi_{t+1}+r_{c, t+1}}\right)=1
$$

To obtain the approximate form of $r_{c, t+1}$, I introduce exogenous processes for consumption growth and inflation. I assume that the following stochastic processes 
for consumption growth and inflation contain small and persistent predictable components.

$$
\begin{aligned}
\left(\begin{array}{c}
g_{c, t+1} \\
\pi_{t+1}
\end{array}\right) & =\mu+X_{t}+\Sigma \eta_{t+1}, \quad \Sigma=\left(\begin{array}{cc}
\sigma_{1} & 0 \\
0 & \sigma_{2}
\end{array}\right) \\
X_{t+1} & =\rho X_{t}+\Phi \Sigma e_{t+1}, \quad \Phi=\left(\begin{array}{ll}
\phi_{11} & \phi_{12} \\
\phi_{21} & \phi_{22}
\end{array}\right), \quad \rho=\left(\begin{array}{ll}
\rho_{11} & \rho_{12} \\
\rho_{21} & \rho_{22}
\end{array}\right) \\
\left(\begin{array}{c}
\eta_{t+1} \\
e_{t+1}
\end{array}\right) & \sim \operatorname{iid\mathcal {N}}(0, I)
\end{aligned}
$$

$\rho$ and $\Phi$ describe the persistence and the conditional volatility of long run risk components $X_{t}$. Therefore, a certain configuration of these parameters may imply that long run risk components are highly persistent and have low volatility. For example, $\rho_{1,1}$ close to 1 and $\phi_{1,1}$ and $\phi_{1,2}$ close to 0 implies that consumption growth contains a small and highly persistent component.

Bansal and Shaliastovich (2007) incorporate the inflation process into a long run risk model to explain nominal bond yields. The above model is a simplified version of their model. ${ }^{1}$ In their model, the volatility of consumption growth is time-varying and consumption risk affects expected inflation and current inflation. Even though such a rich structure may contribute to better term structure implications, there is trade-off because the estimation of the model with time varying volatility demands numerically intensive nonlinear filtering. Another feature that the above model departs from Bansal and Shaliastovich (2007) is that the long run inflation risk component may affect consumption growth through $\rho_{12}$ and $\phi_{12}$. In their model, this channel does not exist and inflation process has no effect on the real economy. In my setup, that result can be empirically tested by estimating $\rho_{12}$ and $\phi_{12}$. I will discuss this point later in the empirical analysis using U.S. data.

\footnotetext{
${ }^{1}$ This specification is also related with Piazzesi and Schneider (2006). The main difference is that I distinguish short run risks $\left(\eta_{t+1}\right)$ from innovations to long run risks $\left(e_{t+1}\right)$. All the risks in their model have long run impacts by the assumption of $\eta_{t+1}=e_{t+1}$.
} 


\subsection{Equilibrium Bond Yields}

What would be the return on consumption claims when the consumption growth and inflation evolve according to the stochastic processes in the above model? In general, this problem does not have a closed form expression. But following Bansal and Yaron (2004), I can obtain the following approximate return on consumption claims by adding the $\log$ price-consumption ratio $\left(z_{t}=\log \left(P_{t} / C_{t}\right)\right)$ as another endogenous variable. $^{2}$

$$
r_{c, t+1}=\kappa_{0}+\kappa_{1} z_{t+1}-z_{t}+g_{c, t+1}, z_{t}=A_{0}+A_{1} X_{t}
$$

As inflation risk factor $\left(x_{2, t}\right)$ affects real economy because it helps predicting the long run consumption risk $\left(x_{1, t+1}\right)$ in the case of $\rho_{12} \neq 0$. By the same reason, the price-consumption ratio is also affected by inflation risk factor. As mentioned by Bansal, Kiku, and Yaron (2007), $\kappa_{0}$ and $\kappa_{1}$ are constants determined by the mean price-consumption ratio $\bar{z}$.

$$
\kappa_{1}=\frac{\exp (\bar{z})}{1+\exp (\bar{z})}, \kappa_{0}=\ln (1+\exp (\bar{z}))-\kappa_{1} \bar{z}
$$

Then we first plug the equation (4) into the equation (3) and obtain three restrictions for $A_{0}$ and $A_{1}$. That would give us $A_{0}$ and $A_{1}$ as functions of $\bar{z}$ and parameters in preference and shock processes. Then $\bar{z}$ can be found numerically by solving a fixed point problem which is $\bar{z}=A_{0}(\bar{z})$. Once this solution is found, the price-consumption ratio and the return on consumption claims are determined. Given $\kappa_{0}$ and $\kappa_{1}, A_{0}$ and $A_{1}$ are determined as follows. ${ }^{3}$

\footnotetext{
${ }^{2}$ The accuracy of this approximation turns out to be reasonably good as discussed in Bansal, Kiku, and Yaron (2007).

${ }^{3}$ The appendix provides the detailed discussion of the derivation of these expressions.
} 


$$
\begin{aligned}
A_{0} & =\frac{\ln \delta+\left(1-\frac{1}{\psi}\right) \mu_{1}+\kappa_{0}+\frac{\theta}{2}\left(\kappa_{1}^{2}\left(\left(A_{11}^{2} \phi_{11}^{2}+A_{12}^{2} \phi_{21}^{2}\right) \sigma_{1}^{2}+\left(A_{11}^{2} \phi_{12}^{2}+A_{12}^{2} \phi_{22}^{2}\right) \sigma_{2}^{2}\right)+(1-\gamma)\left(1-\frac{1}{\psi}\right) \sigma_{1}^{2}\right)}{1-\kappa_{1}} \\
A_{11} & =\frac{\left(1-\kappa_{1} \rho_{22}\right)\left(1-\frac{1}{\psi}\right)}{\kappa_{1}^{2}\left(\rho_{11} \rho_{22}-\rho_{12} \rho_{21}\right)-\left(\rho_{11}+\rho_{22}\right) \kappa_{1}+1} \\
A_{12} & =\frac{\kappa_{1} \rho_{12}\left(1-\frac{1}{\psi}\right)}{\kappa_{1}^{2}\left(\rho_{11} \rho_{22}-\rho_{12} \rho_{21}\right)-\left(\rho_{11}+\rho_{22}\right) \kappa_{1}+1}
\end{aligned}
$$

Armed with the approximate return on consumption claims, we can express the negative log-stochastic discount factor in terms of long-run risk components and their innovations:

$$
\begin{aligned}
-m_{t+1} & =\Gamma_{0}+\Gamma_{1}^{\prime} x_{t}+\Lambda^{\prime} \zeta_{t+1} \\
\zeta_{t+1} & =\left[\sigma_{1} \eta_{1, t+1}, \sigma_{2} \eta_{2, t+1}, \sigma_{1} e_{1, t+1}, \sigma_{2} e_{2, t+1}\right]^{\prime}
\end{aligned}
$$

where $\Gamma_{1}$, and $\Lambda$ are factor loadings and market price of risks, respectively. Expressions for $\Gamma_{1}$ and $\Lambda$ are given by

$$
\begin{aligned}
\Gamma_{0} & =-\theta \ln \delta+\frac{\theta}{\psi} \mu_{1}+(1-\theta)\left(\kappa_{0}+\kappa_{1} A_{0}-A_{0}+\mu_{1}\right)+\mu_{2} \\
\Gamma_{1} & =\left[\frac{1}{\psi}, 1\right]^{\prime} \\
\Lambda & =\left[\gamma, 1,(1-\theta) \kappa_{1}\left(A_{11} \phi_{11}+A_{12} \phi_{21}\right),(1-\theta) \kappa_{1}\left(A_{11} \phi_{12}+A_{12} \phi_{22}\right)\right]^{\prime}
\end{aligned}
$$

Given the log nominal stochastic discount factor, we can compute arbitrage-free nominal bond prices from Euler equations. This calculation can be done relatively easily because the nominal stochastic discount factor follows a log-normal distribution.

$$
e^{p_{n, t}}=E_{t}\left(e^{m_{t+1}+p_{n-1, t+1}}\right) \Longrightarrow p_{n, t}=E_{t}\left(m_{t+1}+p_{n-1, t+1}\right)+\frac{V_{t}\left(m_{t+1}+p_{n-1, t+1}\right)}{2}
$$


where $p_{n, t}$ is the log of the price of a nominal bond whose time to maturity is $n$ period. Under homoskedasticity, the above equation implies $p_{n, t}=E_{t}\left(\sum_{j=1}^{n} m_{t+j}\right)+$ $\frac{V_{t}\left(\sum_{j=1}^{n} m_{t+j}\right)}{2} .{ }^{4}$ Since $m_{t+1}$ is affine with respect to risk factors, we can express $p_{n, t}$ as an affine function of risk factors $\left(A_{n}+B_{n} x_{t}\right)$ as well. Now the model implied bond yields are also affine functions of risk factors by the following relation:

$$
y_{n, t}=-\frac{p_{n, t}}{n}=a_{n}+b_{n} x_{t} \Longrightarrow y_{n, t}=\frac{E_{t}\left(\sum_{j=1}^{n}-m_{t+j}\right)-0.5 V_{t}\left(\sum_{j=1}^{n} m_{t+j}\right)}{n}
$$

\subsection{Unconditional Expected Excess Return for Long Term Bonds}

Piazzesi and Schneider (2006) show that the negative correlation between inflation and consumption is crucial in generating a positive expected excess return for long term bonds. Since they consider a special case in which the intertermporal elasticity of substitution is 1 , it would be useful to check their results can be generalized in our setting. Here, I consider the unconditional expected excess return which can be defined as follows:

$$
E\left(r x_{t+1}^{n}\right)=E\left(p_{n-1, t+1}-p_{n, t}-y_{1, t}\right)=-E\left[\operatorname{cov}_{t}\left(m_{t+1}, p_{n-1, t+1}\right)+0.5 V_{t}\left(p_{n-1, t+1}\right)\right]
$$

Since $V_{t}\left(p_{n-1, t+1}\right)$ is always nonnegative, we can consider $-E\left[\operatorname{cov}_{t}\left(m_{t+1}, p_{n-1, t+1}\right)\right]$ as an upper bound for the unconditional expected excess return for a $n$ period bond. The unconditional expectation of this covariance term depends explicitly on correlation parameters such as $\rho_{12}, \rho_{21}, \phi_{12}, \phi_{21}$. For example, the upper bound for the unconditional expected excess return of a 2 period bond is given by

$\sigma_{1}^{2}\left[(1-\theta) \kappa_{1}\left(A_{11} \phi_{11}+A_{12} \phi_{21}\right)\left(\frac{\phi_{11}}{\psi}+\phi_{21}\right)\right]+\sigma_{2}^{2}\left[(1-\theta) \kappa_{1}\left(A_{11} \phi_{12}+A_{12} \phi_{22}\right)\left(\frac{\phi_{12}}{\psi}+\phi_{22}\right)\right]$

\footnotetext{
${ }^{4}$ The homoskedasticity assumption is needed to guarantee not only $m_{t+1}$ but also $\sum_{j=1}^{n} m_{t+j}$ is normally distributed.
} 
Let's consider two special cases. Suppose $\theta$ is 1 , which corresponds to the expected utility case, then the upper bound of the unconditional expected excess return is 0 . Also, if inflation and consumption are not correlated $\left(\rho_{12}=\rho_{21}=\phi_{12}=\right.$

$\left.\phi_{21}=0\right)$, the upper bound is $\sigma_{1}^{2} \frac{(1-\gamma) \kappa_{1} \phi_{11}^{2}}{\left(1-\kappa_{1} \rho_{11}\right) \psi}$. Therefore, the upper bound is negative if $\gamma \geq 1$. The above expression suggests that preference parameters and parameters governing shock processes interact in a delicate way and we need a certain configuration of parameters in order to generate a positive average excess return. The negative correlation of consumption and inflation may help us to generate a positive average excess return for some values of preference parameters. Therefore, using term structure data to estimate $\rho$ and $\phi$ together with preference parameters can be important to uncover term structure implications of long run risk models.

\section{$3 \quad$ Econometric Methodology}

With data on consumption growth, inflation, and bond yields available $\left(Y_{t}=\right.$ $\left.\left[g_{c, t}, \pi_{t}, y_{1, t}, \cdots, y_{n, t}\right]^{\prime}\right)$, I can estimate the model based on the likelihood constructed by Kalman filtering. Indeed, the above model is a special case of a linear Gaussian state space model in which long run risk components play the role of latent states.

$$
\begin{aligned}
S_{t} & =\rho_{S}(\vartheta) S_{t-1}+\Phi_{S}(\vartheta) \Sigma(\vartheta) e_{t} \\
Y_{t} & =a_{Y}(\vartheta)+b_{Y}(\vartheta) S_{t}+c_{Y}(\vartheta) \xi_{t} \\
\vartheta & =\left[\rho_{11}, \rho_{12}, \rho_{21}, \rho_{22}, \phi_{11}, \phi_{21}, \phi_{22}, \sigma_{1}, \sigma_{2}, \mu_{1}, \mu_{2}, \beta, \psi, \gamma\right]^{\prime} \\
\xi_{t} & =\left[\eta_{1, t}, \eta_{2, t}, u_{1, t}, \cdots, u_{n, t}\right]^{\prime}, u_{i, t} \sim i . i . d . \mathcal{N}\left(0, \sigma_{u}^{2}\right)
\end{aligned}
$$

where $S_{t}=\left[X_{t}^{\prime}, X_{t-1}\right]^{\prime}$ is a vector of augmented state variables and $\xi_{t}$ is a vector of measurement errors for observed variables and $\vartheta$ is a vector of structural parameters governing preference and shock processes. We introduce measurement errors to bond yields in order to avoid the singularity problem. In this setting, short run risks in consumption and inflation are observationally equivalent to measurement 
errors in consumption growth and inflation. I fix the value $\sigma_{u}$ to roughly $9 \%$ of the standard deviation of bond yields.

The likelihood function in the state space model is given by decomposing the prediction error as follows:

$$
\begin{aligned}
\mathcal{L}\left(\vartheta \mid Y^{T}\right) & =\prod_{t=1}^{T} p\left(Y_{t} \mid Y^{t-1}, \vartheta\right)=\prod_{t=1}^{T} \int p\left(Y_{t} \mid S_{t}, \vartheta\right) p\left(S_{t} \mid Y^{t-1}, \vartheta\right) d S_{t} \\
Y^{t} & =\left[Y_{1}, \cdots, Y_{t}\right], Y_{0}=\{\}
\end{aligned}
$$

In the linear Gaussian case, Kalman filtering provides the analytical solution for the integration problem and we can evaluate the likelihood without approximation of integration appearing in equation (15). ${ }^{5}$ I restrict my attention to the area of parameter space which guarantees the stationarity of $X_{t}$ to rule out non-stationarity in consumption growth or inflation.

Since the long run risk components are not directly observed, we may be interested in backing out the estimates of them given all the available observations $Y^{T}$. Running Kalman filtering backwards, I obtain smoothed estimates of the state variables conditional on $Y^{T}$, which are given by

$$
\widehat{S_{t}^{\vartheta}}=E\left(S_{t} \mid Y^{T}, \vartheta\right)
$$

Once the likelihood function is evaluated by Kalman filtering, I can obtain the maximum likelihood estimates of parameters. I take Bayesian approach and combine the likelihood with the prior density of parameters. There are some advantages of using Bayesian approach in this context. First, system matrices in measurement equations of the state space model $\left(a_{Y}(\vartheta)\right.$ and $\left.b_{Y}(\vartheta)\right)$ are highly nonlinear with respect to parameters because they depend on the solution of a nonlinear equation for the mean price-consumption ratio. The solution of this nonlinear equation is

\footnotetext{
${ }^{5}$ When we allow stochastic volatility terms, the model cannot be written as a linear Gaussian state space form and the evaluation of the integration demands numerically intensive methods such as particle filtering.
} 
found by numerical methods. The use of numerical solution creates the possibility that the likelihood may not be smooth along the dimension of some parameters and makes it difficult to obtain confidence intervals for estimates of parameters. ${ }^{6}$ In Bayesian inference, the posterior probability intervals can still be obtained as long as data is informative about these parameters and the likelihood does not have fat tails. ${ }^{7}$ Second, the existing literature on long risks models has some information about parameter values which are in line with stylized facts of asset market data. Since I use only term structure data in the estimation, I would like to put less emphasis on parameter values which are incongruent with stock market data.

When the likelihood is combined with the prior distribution of $\vartheta$, the posterior distribution of $\vartheta$ is given by

$$
p\left(\vartheta \mid Y^{T}\right)=\frac{\mathcal{L}\left(\vartheta \mid Y^{T}\right) p(\vartheta)}{p\left(Y^{T}\right)}
$$

The posterior density of $\vartheta$ is complicated and does not have a known closed form. So I rely on simulation methods to compute posterior moments of parameters and use Markov Chain Monte Carlo methods described in Schorfheide (2000). ${ }^{8}$ The algorithm to implement simulation consists of two steps. First, I generate 100,000 draws from the prior distribution of parameters and eliminate draws implying nosstationary $X_{t}$. Then I select one draw with the highest likelihood value among 1000 draws picked in the prior draws. ${ }^{9}$ Second, using the selected draw as a starting point, I generate 200,000 draws by applying a Metropolis-Hastings algorithm initialized around that point. Then I discard the first 50,000 draws to attenuate the dependence

\footnotetext{
${ }^{6}$ This concern turns out to be relevant for parameters governing correlation between consumption growth and inflation such as $\rho_{12}, \rho_{21}, \phi_{12}, \phi_{21}$.

${ }^{7}$ This is to say that conditions for the convergence of MCMC chains are satisfied and there are no identification issues for parameters. See Geweke (2005) for precise conditions necessary for the convergence of Markov chains. However, asymptotic distribution of posterior moments would be difficult to characterize in the presence of the non-smooth likelihood function.

${ }^{8}$ Details of this procedure are provided in the appendix.

${ }^{9}$ This strategy of selecting starting points for Markov chains is borrowed from Justiniano and Primiceri (2008).
} 
on the starting point. I compute posterior moments based on the remaining 150,000 draws.

We can also evaluate the fit of different models based on marginal data densities as follows:

$$
p\left(Y^{T} \mid \mathcal{M}_{i}\right)=\int \mathcal{L}\left(\vartheta \mid Y^{T}, \mathcal{M}_{i}\right) p\left(\vartheta \mid \mathcal{M}_{i}\right) d \vartheta
$$

\section{Empirical Analysis}

Now, I turn to empirical analysis of the model using U.S. data. The main focus is to extract estimates of long run risk components from the term structure of interest rates data.

\subsection{Data}

I use the same dataset as the one in Piazzesi and Schneider (2006) except for the slight change in the sample period. ${ }^{10}$ Aggregate consumption growth is from the quarterly NIPA data on nondurables and services. Following Piazzesi and Schneider (2006), I use the price index for NIPA data. 3 month treasury bill rate from the CRSP Fama riskfree rate file is used as data for the short term interest rate. One, two, three, four, five year bond yields are extracted from the CRSP Fama-Bliss discount bond files.

\subsection{Prior Distribution of Parameters}

There are two sets of parameters in the model. For one set of parameters related to the stochastic processes for consumption growth and inflation, I set prior distributions of parameters to be roughly consistent with i) sample moments of consumption

\footnotetext{
${ }^{10}$ The sample period in Piazzesi and Schneider (2006) is from 1952:Q2 to 2005:Q4 while it is from 1953:Q1 to 2006:Q4 in my case.
} 
growth and inflation and ii) calibrated values in the literature on long run risk models. For preference parameters, the prior mean values are set to be close to estimated values of those parameters in the existing literature.

The prior mean values of $\rho_{11}$ and $\rho_{22}$ are set to be 0.92 . Comparable parameter values used in Bansal and Shaliastovich (2007) are 0.991 and 0.83 for $\rho_{11}$ and $\rho_{22}$, respectively. These values are within 2 standard deviation in the prior distribution. Constructing the prior distribution of $\rho_{12}$ and $\rho_{21}$ is more difficult because the existing literature imply a variety of estimates even with different signs. The point estimates of $\rho_{12}$ and $\rho_{21}$ in Piazzesi and Schneider (2006) are -0.099 and 0.28. In contrast, Bansal and Shaliastovich (2007) set $\rho_{12}$ to 0 to reflect their belief that inflation does not affect the real activity while they assume a negative value for $\rho_{21}$ so that low consumption growth predicts high inflation. The empirical relevance of the real impacts of inflation factor can be tested by comparing the marginal data density of two models with different priors for the cross correlation structure of consumption and inflation. To shut down the impacts of inflation factor on real activity, I fix parameters values for $\rho_{12}$ and $\phi_{12}$ to 0 in one set of prior. In another set of prior, I allow for variation of $\rho_{12}$ and $\phi_{12}$ around 0 .

I calibrate the prior mean of $\phi_{11}$ and $\phi_{22}$ so that the implied autocorrelations of consumption growth and inflation at the prior mean of $\rho$ and $\Phi$ are equal to sample moments. This calibration is especially important for consumption growth because observed consumption growth does not show high persistence even though we allow a fairly persistent long run risk component. I also set the standard deviations of short run risks to match the implied standard deviations of consumption growth and inflation with sample moments. Prior mean values of average consumption growth and inflation are set to be 0.007 which is a bit lower than sample moments. However, together with the prior mean of preference parameters, these values help to match the implied average short term rate with the sample mean of short term rate.

For preference parameters $\delta, \psi$, and $\gamma$, I use the information from the existing estimation results to construct the prior distribution. In Chen, Favilukis, and Ludvigson (2007), the point estimates $\delta, \psi$, and $\gamma$ are 0.999, 2, and 60. ${ }^{11}$ In Bansal,

\footnotetext{
${ }^{11}$ This is the case when they aggregate consumption data and the inverse of covariance matrix
} 
Kiku, and Yaron (2007), the point estimates of $\psi$ and $\gamma$ are 2.43 and 10.28 with their standard deviations 1.33 and 1.99. They fix the discount factor to 0.987 . I set the prior distribution of $\beta$ and $\psi$ so that the prior distribution can be compatible with both estimates. Table 1 summarizes the prior information for all the parameters.

\subsection{Posterior Analysis}

\subsubsection{Posterior Distribution}

We can revise our prior belief about parameters by using the information from data. Table 2 illustrates how the data refines our belief about parameters by contrasting prior distribution with posterior distribution. For persistence parameters of the long run risk factors, the posterior intervals are much smaller than prior intervals, indicating there is much information from data about these parameters. In addition, the posterior mean values of some parameters are quite different prior mean values. For example, the posterior distribution of $\rho_{21}$ centers around 0.361 in contrast to the prior mean value of 0 . On the other hand, the posterior mean of $\rho_{12}$ is negative, which is consistent with the view that high inflation predicts low consumption growth as emphasized by Piazzesi and Schneider (2006). When I shut down the real impacts of inflation factor, $\rho_{21}$ is estimated to be significantly negative. This result indicates that low consumption growth predicts high inflation. Both models are compatible with the observed negative correlation of consumption and inflation and thus, we need another criterion to evaluate the relative fit of the two models. The log marginal data density of Table 3 shows that data strongly favors the real impacts of inflation factor.

Compared to the prior distribution, the posterior probability interval of discount factor $(\beta)$ is much smaller. In addition the posterior mean of the intertemporal elasticity of substitution $(\psi)$ is higher than the prior mean. The data does not reveal much additional information about the risk aversion parameter $(\gamma)$. In the existing literature, the estimated value of risk aversion is typically greater than 6 (even higher than 20 in some cases of Chen, Favilukis, and Ludvigson (2007)) and the as a weighting matrix in the estimation. 
estimate of the intertemporal elasticity of substitution is greater than 1 . When we allow time varying volatility of shocks, the condition of $\gamma>1>\frac{1}{\psi}$ guarantees that investors dislike the rising uncertainty and demand a risk premium. ${ }^{12}$ The estimates here are compatible with this condition for parameters. Additionally, I check the role of recursive preferences in explaining term structure data by comparing the log marginal data density of the benchmark model with that of the expected utility version. Table 3 shows that the data supports recursive preferences. ${ }^{13}$ In total, the model needs both the generalized correlation of long run risk components and recursive preferences to fit term structure data better.

\subsubsection{Macro Implications}

Now I compare model implied posterior moments for macro variables with sample moments from data to explore the model's macro implications. One complication in computing posterior moments is that they are functions of parameters and the latent state variables. The posterior distribution of latent state variables conditional on parameters can be obtained by running Kalman smoothing. Smoothed estimates of latent state variables can then be used to compute posterior moments.

Table 4 provides information about the model's implications for dynamics of consumption growth and inflation. The model matches the mean and volatility of consumption growth well although the first order autocorrelation implied by the model is a bit lower than the sample counterpart. Regarding inflation dynamics, the model does not perform well. The model implied mean and volatility of inflation are much higher than sample counterparts while autocorrelations are lower than suggested by data. The model implied correlation of consumption growth and inflation is negative, which is consistent with data. However, the magnitude is much smaller than we observe in sample data. This results indicates our specification for shock processes may be severely misspecified. To check this possibility, I estimate

\footnotetext{
${ }^{12}$ This point is emphasized in Bansal and Yaron (2004).

${ }^{13}$ According to a widely used criterion of model comparison described in Jeffreys (1961), the posterior odds higher than 32 indicates "very strong" or "decisive" conclusion. In terms of log marginal data density, this is equivalent to 3.4657 .
} 
the same processes with data only on consumption and inflation. In this case, the posterior moments in macro estimation now track the sample counterparts well. Therefore, I infer that there is some tension in tracking inflation dynamics and the time series of term structure data well at the same time. Figure 1 illustrates the problem. Inflation rapidly rose during the mid 1970s and fell quickly during the early 1980s. Also, consumption growth moved in the opposite direction during two episodes, amplifying the negative correlation between consumption growth and inflation. However, interest rates rose only mildly during the mid 1970s and declined rather slowly during the 1980s. In the joint estimation, consumption growth and inflation are risk factors whose behavior should be consistent with changes in interest rates. That constraint creates the tension we observe in the joint estimation. ${ }^{14}$ Indeed, the short term interest rate during the mid 1980s is associated with the Fed's aggressive response to the inflation scare of 1983-84 as pointed by Goodfriend (1993). For that reason, interest rates data shows the striking deviation from the macro-based rule. That fact creates a difficulty even in a Taylor-rule based noarbitrage term structure model such as Bikbov and Chernov (2006) even if they use an affine pricing kernel not restricted by a particular type of preference.

Figure 2 and 3 show estimates of the long run risk components. Consistent with the low autocorrelation of measured consumption growth, the long run risk component in consumption growth is fairly small in terms of volatility. In contrast, the long run risk component in inflation is as volatile as the measured inflation, which reflects the relatively high persistence of inflation data. Also, the long run risk component in inflation varies a lot depending on which dataset we use. One alternative to backing out latent long run components by Kalman filtering is to regress observed consumption growth or inflation on the previous period's term structure variables. Bansal, Kiku, and Yaron (2007) take this approach and extract expected consumption growth from the regression of the current period consumption on the

\footnotetext{
${ }^{14}$ Doh (2007) finds a portion of this discrepancy from the imperfect information of private agents about changing inflation target of the central bank as modelled in Erceg and Levin (2003). However, it is not enough to reconcile term structure data with inflation data at the quantitatively significant level.
} 
risk-free rate and market price dividend ratio. Here, I regress consumption growth on a constant and the 6 bond yields used in the estimation. Figure 4 shows estimates of long run consumption risk component extracted by the regression. The regression based estimates of long run consumption risk imply lower autocorrelation and higher predictability of consumption growth than filtered estimates. Table 5 shows the autocorrelation of regression based estimates is 0.634 and $R^{2}$ in the regression of consumption growth on a constant and the estimate of long run consumption risk is 0.127 while corresponding numbers for the filtered estimates are 0.888 and 0.031, respectively. Given the importance of the persistence parameter in long run risk models, this comparison gives some caution against using the regression-based estimates of latent long run factors to explore the model's asset pricing implications.

\subsubsection{Term Structure Implications}

Now, I compare the posterior moments of the yield curve implied by the estimated model with their sample counterparts in the data. I focus on mean and volatility of term structure data and set aside the empirical failure of expectations hypothesis which is discussed in Bansal and Shaliastovich (2007). This is due to the restriction that I do not have time-varying volatility which can account for the empirical failure of expectations hypothesis. Table 6 provides information on term structure implications of the model. The estimated model can do a relatively good job in matching first and second moments of the yield curve. It also matches well the high persistence of bond yields. In a comparable model, Piazzesi and Schneider (2006) report the difficulty of matching the volatility of the yield curve without introducing additional amplification mechanism such as learning. In particular, the model implied volatility of long term rates is much lower and less than a half of sample standard deviation in the benchmark model of Piazzesi and Schneider (2006). The difference can be understood by realizing that we use term structure data to extract long run risk components while they use only macro data to estimate these components. Since the implied volatility of inflation is higher in the joint estimation and inflation contains a persistent component, it contributes to high volatility of long term rates. 
Another measure to evaluate the model's in-sample fit for term structure variables is the ex-post measurement error. Table 6 provides more information on those measurement errors. Indeed, the estimated model captures the time variation of long term rates quite well. For example, the mean absolute value of ex-post measurement error for a 5 year bond yield is roughly 8 basis point. Overall, the magnitude of measurement errors for bond yields is smaller than the standard deviation of those errors fixed at 24 basis points. Also except for a 1 year bond yield, the magnitude of ex-post measurement error is smaller than comparable numbers reported in Bikbov and Chernov (2006), who report average pricing errors between about 9 basis points and 28 basis points. While they incorporate monetary policy rule in a no-arbitrage framework, their pricing kernel is exogenously given unlike the estimated model in this paper where the pricing kernel is tied down by the recursive preference. Also, the estimated long run risk model here provides clear economic interpretation for latent state variables $X_{t}$ while Bikbov and Chernov (2006) demand additional work to clarify economic meaning of latent term structure variables.

\subsection{Robustness}

Cochrane (2007) expresses skepticism of explaining the movement of aggregate asset prices based on long-run properties of consumption growth. He argues that Bansal and Yaron (2004) do not provide strong direct evidence for the required long-run properties of consumption growth and without independent measurements, movements in long-run consumption risk may just reflect unobserved shifts in marginal utility. In response to the criticism, this paper provides direct evidence for long run risk components in consumption growth and inflation by filtering these components from the term structure data. One possible objection to results suggested here is that I use a fairly concentrated prior for the persistence parameter for consumption growth $\left(\rho_{11}\right)$ and this may tilt estimates in favor of the existence of long run risks. To make sure that this is not the case, I estimate the above model with a different

prior on $\rho_{11}, \phi_{11}$, and $\sigma_{1}^{2}$. Again, I set prior mean values of these parameters to match the model implied autocorrelation and standard deviation of consumption growth with sample counterparts. 
When the term structure data is used for the estimation, the posterior distribution of the persistence parameter of the long run consumption risk is still high. However, when only macro data is used, the persistence parameter actually shifts downward. Table 7 provides the detailed information about the estimation results. Also, Table 3 shows log marginal data density for each case. In the macro estimation, the low serial correlation of expected consumption growth is favored by data while a persistent process for expected consumption growth is supported in joint estimation. The finding provides a caution against estimating parameters of macro dynamics with only macro data and plugging these estimates to derive equilibrium asset prices.

\section{Conclusion}

In this paper, I estimate a long run risk model by using the term structure of nominal interest rates data. With the combination of Epstein-Zin (1989) recursive preferences and general correlation structure between inflation and consumption growth, the long run risk model captures the time variation of the nominal term structure data well, especially in the long end of the yield curve. In particular, the paper shows that real impacts of inflation factor and the existence of a small but persistent component in consumption growth are very important.

Although long run risk models can explain many stylized facts in a parsimonious way (e.g. Bansal and Yaron (2004), Bansal and Shaliastovich (2007)), direct estimates of long run risk components are rare, or their latent structure is not fully utilized in the estimation (e.g. Bansal, Kiku, and Yaron (2007)). In this paper, I filter out short run risks to obtain estimates of latent long run risk components from observed data on the yield curve. Smoothed estimates of long run consumption growth component are much less volatile but more persistent than observed consumption growth data. The evidence is in line with the key assumption of long run risk models. 


\section{Appendix}

\subsection{The Approximation of the Return on Consumption Claims}

The Euler equation for claims on consumption implies the following restriction.

$$
E_{t}\left(e^{m_{t+1}+\pi_{t+1}+r_{c, t+1}}\right)=E_{t}\left(e^{\theta \log \delta-\frac{\theta}{\psi} g_{c, t+1}+\theta r_{c, t+1}}\right)=1
$$

Under the linear approximation of $r_{c, t+1}$ and the conditional normality of shocks, we can rewrite equation (20) into

$$
E_{t}\left(e^{m_{t+1}+\pi_{t+1}+r_{c, t+1}}\right)+\frac{V_{t}\left(e^{m_{t+1}+\pi_{t+1}+r_{c, t+1}}\right)}{2}=0
$$

This implies the three restrictions on $A_{0}, A_{11}, A_{12}$.

$$
\begin{aligned}
-\frac{\theta}{\psi}+\theta\left[\kappa_{1}\left(A_{11} \rho_{11}+A_{12} \rho_{21}\right)-A_{11}+1\right]= & 0 \\
\theta\left[\kappa_{1}\left(A_{11} \rho_{12}+A_{12} \rho_{22}\right)-A_{12}\right]= & 0 \\
\theta \ln \delta+\left(\theta-\frac{\theta}{\psi}\right) \mu_{1}+\theta\left(\kappa_{0}+\kappa_{1} A_{0}-A_{0}\right)= & -\frac{\theta^{2}}{2}\left[\kappa_{1}^{2}\left(\left(A_{11}^{2} \phi_{11}^{2}+A_{12}^{2} \phi_{21}^{2}\right) \sigma_{1}^{2}+\left(A_{11}^{2} \phi_{12}^{2}+A_{12}^{2} \phi_{22}^{2}\right) \sigma_{2}^{2}\right)\right. \\
& \left.+(1-\gamma)\left(1-\frac{1}{\psi}\right) \sigma_{1}^{2}\right]
\end{aligned}
$$

Equation (6) can be obtained from the above restrictions.

\subsection{Markov Chain Monte Carlo Methods for Posterior Simulation}

The following Metropolis Hastings algorithm is used in the estimation.

- Step 1 Selection of the Starting Point : Compute the log-likelihood for 100 draws from prior distribution. Select one point which gives the highest $\log$-likelihood value $\vartheta^{\star}$. 
- Step 2 Proposal : Starting from $\vartheta^{\star}$, generate a new draw by the following proposal density, which is a mixture of normal and $t$ density. The transition mixture allows the occasional jump to a heavy-tailed distribution and is useful for exploring deeply separated areas of parameter space with roughly similar posterior density (Geweke (2005, pp. 142-3)). The scaling matrix $c$ is chosen by multiplying a small positive real number to the prior covariance matrix.

$$
\vartheta^{N, j+1}=\vartheta^{j}+c[p \mathcal{N}(0, I)+(1-p) t(0, I, s)], j=0, \cdots, R-1
$$

Here, $p$ is mixing probability ( 0.9 is used for the estimation) and $s$ is the degree of freedom in the multivariate $t$ distribution. $s$ is set to 5 in the estimation.

- Step 3 Accept/Reject Compute the acceptance rate $\alpha=\min \left\{\frac{p\left(\vartheta^{N, j+1} \mid z^{T}\right)}{p\left(\vartheta^{j} \mid z^{T}\right)}, 1\right\}$ and accept or reject $\vartheta^{N, j+1}$ according to the value of $u$ which is drawn from the uniform distribution over the unit interval $[0,1]$.

$$
\begin{gathered}
\vartheta^{j+1}=\vartheta^{N, j+1}, \text { if } u<\alpha \\
\vartheta^{j}, \text { otherwise }
\end{gathered}
$$

- Step 4 Burn-In For the purpose of the posterior inference, burn in the initial $B(50,000$ in the estimation) draws and use the remaining draws for posterior inference.

\section{References}

Bansal, R., R. Gallant, and G. Tauchen (2007): "Rational Pessimism, Rational Exuberance, and the Cross-Section of Equity Returns, "Review of Economic Studies, 74(4), 1005-33.

Bansal, R. ,D. Kiku, and A. Yaron (2007): "Risks for the Long Run: Estimation and Inference, "Manuscript, Duke University. 
Bansal, R. and I. Shaliastovich (2007): "Risk and Return in Bond, Currency, and Equity Markets," Manuscript, Duke University.

Bansal, R. and A. Yaron (2004): "Risks for the Long Run: A Potential Resolution of Asset Pricing Puzzles," Journal of Finance, 59, 1481-1509.

Bikbov, R. and M. Chernov (2006): "No-Arbitrage Macroeconomic Determinants of the Yield Curve," Manuscript, Columbia University.

Chen, X., J. Favilukis, and S.Ludvigson (2007): "An Estimation of Economic Models with Recursive Preferences, " Manuscript, New York University.

Cochrane, J. (2007): "Financial Markets and the Real Economy, "Manuscript, University of Chicago.

Doh, T. (2007): "What does the Yield Curve Tell us about the Federal Reserve's Implicit Inflation Target?, "Manuscript, Federal Reserve Bank of Kansas City.

Epstein, L. and S. Zin (1989): "Substitution, Risk Aversion and the Temporal Behavior of Consumption and Asset Returns: A Theoretical Framework, "Econometrica, $\mathbf{5 7}(4), 937-69$.

Erceg, C.J.,A.T. Levin (2003): "Imperfect Credibility and Inflation Persistence," Journal of Monetary Economics, 50(4), 915-44.

Geweke, J. (2005): Contemporary Bayesian Econometrics and Statistics, Hoboken,NJ: John Wiley \& Sons.

Goodfriend, M. (1993): "Interest Rate Policy and Inflation Scare Problem:19791992," Federal Reserve Bank of Richmond Economic Quarterly 79(1), 1-23.

Jeffreys, H. (1961): Theory of Probability, Oxford: Oxford University Press.

Justiniano, A. and G.E. Primiceri (2008): "The Time Varying Volatility of Macroeconomic Fluctuations," American Economic Review, forthcoming.

Newey, W. K., and K. D. West (1987): "A Simple, Positive Semi-definite, Heteroskedasticity and Autocorrelation Consistent Covariance Matrix," Econometrica $\mathbf{5 5}(3), 703-8$. 
Piazzesi, M. and M. Schneider (2006): "Equilibrium Bond Yields," NBER Macroeconomics Annual, edited by Daron Acemoglu, Kenneth Rogoff and Michael Woodford, Cambridge,MA:MIT Press.

Schorfheide, F. (2000): "Loss Function-Based Evaluation of DSGE Models," Journal of Applied Econometrics, 15, 645-670. 
Table 1: Prior Distribution

\begin{tabular}{|c|c|c|c|c|}
\hline Parameters & Domain & Density & Para(1) & $\operatorname{Para}(2)$ \\
\hline \multirow[t]{2}{*}{$\rho_{11}$} & {$[0,1)$} & Beta & 0.92 & 0.05 \\
\hline & {$[0,1), \mathrm{P} 5$} & Beta & 0.4 & 0.1 \\
\hline \multirow[t]{2}{*}{$\rho_{12}$} & $\mathbb{R}$ & Normal & 0 & 0.1 \\
\hline & P2, P3 & & 0 & fixed \\
\hline \multirow[t]{2}{*}{$\rho_{21}$} & $\mathbb{R}$ & Normal & 0 & 0.1 \\
\hline & P3 & & 0 & fixed \\
\hline$\rho_{22}$ & {$[0,1)$} & Beta & 0.92 & 0.05 \\
\hline \multirow[t]{3}{*}{$\phi_{11}$} & $\mathbb{R}^{+}$ & Gamma & 0.3 & 0.05 \\
\hline & $\mathrm{P} 2$ & & 0 & fixed \\
\hline & $\mathbb{R}^{+}, \mathrm{P} 5$ & Gamma & 2.54 & 0.3 \\
\hline \multirow[t]{2}{*}{$\phi_{21}$} & $\mathbb{R}$ & Normal & 0 & 0.05 \\
\hline & $\mathrm{P} 3$ & & 0 & fixed \\
\hline$\phi_{22}$ & $\mathbb{R}^{+}$ & Gamma & 1.26 & 0.15 \\
\hline \multirow[t]{2}{*}{$\sigma_{1}^{2}$} & $\mathbb{R}^{+}$ & Inverse Gamma & 0.000013 & 4 \\
\hline & $\mathbb{R}^{+}, \mathrm{P} 5$ & Inverse Gamma & 0.0000024 & 4 \\
\hline$\sigma_{2}^{2}$ & $\mathbb{R}^{+}$ & Inverse Gamma & 0.0000036 & 4 \\
\hline$\mu_{1}$ & $\mathbb{R}$ & Normal & 0.007 & 0.0002 \\
\hline$\mu_{2}$ & $\mathbb{R}$ & Normal & 0.007 & 0.0002 \\
\hline$\delta$ & {$[0,1)$} & Beta & 0.997 & 0.002 \\
\hline$\psi$ & $\mathbb{R}^{+}$ & Gamma & 1.5 & 0.2 \\
\hline \multirow[t]{2}{*}{$\gamma$} & $\mathbb{R}^{+}$ & Gamma & 17 & 3 \\
\hline & $\mathrm{P} 4$ & & $\frac{1}{\psi}$ & \\
\hline
\end{tabular}

Notes: Para (1) and Para (2) list the means and the standard deviations for Beta, Gamma, and Normal distributions; $s$ and $\nu$ for the Inverse Gamma distribution, where $p_{\mathcal{I G}}(\sigma \mid \nu, s) \propto$ $\sigma^{-\nu-1} e^{-\nu s^{2} / 2 \sigma^{2}}, a$ and $b$ for the Uniform distribution from $a$ to $b$. Standard deviations of measurement errors are fixed as explained in the paper. P2, P3 denote alternative prior distributions in which correlation between consumption growth and inflation is restricted while P4 denotes the expected utility version. P5 reflects the belief that consumption growth does not involve a fairly persistent component. 
Table 2: Posterior Distribution

\begin{tabular}{cccccc}
\hline \hline Parameter & Prior & \multicolumn{3}{c}{ Posterior } \\
\hline & & & P1 & P2 \\
& $90 \%$ Interval & Mean & $90 \%$ Interval & Mean & $90 \%$ Interval \\
\hline$\rho_{11}$ & {$[0.848,0.992]$} & 0.873 & {$[0.853,0.893]$} & 0.870 & {$[0.848,0.889]$} \\
$\rho_{12}$ & {$[-0.165,0.165]$} & -0.006 & {$[-0.008,-0.004]$} & & \\
& 0 & & & & \\
$\rho_{21}$ & {$[-0.163,0.166]$} & 0.361 & {$[0.306,0.422]$} & -0.409 & {$[-0.484,-0.332]$} \\
$\rho_{22}$ & {$[0.849,0.993]$} & 0.997 & {$[0.994,0.999]$} & 0.976 & {$[0.969,0.982]$} \\
$\phi_{11}$ & {$[0.220,0.384]$} & 0.161 & {$[0.133,0.188]$} & 0.136 & {$[0.112,0.162]$} \\
$\phi_{12}$ & {$[-0.081,0.083]$} & -0.126 & {$[-0.153,-0.099]$} & & \\
& 0 & & & & \\
$\phi_{21}$ & {$[-0.083,0.083]$} & -0.069 & {$[-0.127,-0.031]$} & 0.125 & {$[0.066,0.185]$} \\
$\phi_{22}$ & {$[1.011,1.504]$} & 0.626 & {$[0.560,0.687]$} & 0.479 & {$[0.419,0.535]$} \\
$10000 \sigma_{1}^{2}$ & {$[0.069,0.257]$} & 0.192 & {$[0.163,0.223]$} & 0.216 & {$[0.183,0.250]$} \\
$10000 \sigma_{2}^{2}$ & {$[0.019,0.071]$} & 0.268 & {$[0.237,0.305]$} & 0.287 & {$[0.244,0.329]$} \\
$400 \mu_{1}$ & {$[2.88,3.96]$} & 2.94 & {$[2.72,3.14]$} & 3.60 & {$[3.42,3.78]$} \\
$400 \mu_{2}$ & {$[3.24,3.92]$} & 3.78 & {$[3.52,4.06]$} & 3.51 & {$[3.20,3.85]$} \\
$\delta$ & {$[0.9942,0.9998]$} & 0.995 & {$[0.992,0.998]$} & 0.998 & {$[0.997,0.999]$} \\
$\psi$ & {$[1.178,1.829]$} & 1.594 & {$[1.303,1.857]$} & 1.715 & {$[1.407,2.008]$} \\
$\gamma$ & {$[12.134,21.881]$} & 14.616 & {$[11.810,17.382]$} & 10.937 & {$[8.234,13.537]$} \\
\hline & & & & &
\end{tabular}


Table 3: Log Marginal Data Densities

\begin{tabular}{cccccc}
\hline \hline Data & Benchmark & P2 & P3 & P4 & P5 \\
\hline Joint & 8959.7 & 8916.6 & 8836.3 & 8952.9 & 8898.0 \\
Macro & 1719.2 & 1707.5 & 1696.3 & & 1728.9 \\
\hline
\end{tabular}

Notes: P3 stands for the model in which $\rho_{12}, \rho_{21}, \phi_{12}, \phi_{21}$ are fixed at 0 and P4 denotes the model with expected utility in which $\gamma$ is constrained to be equal to $\frac{1}{\psi}$. The change in the preference specification does not affect the macro estimation because preference parameters are not used in the macro estimation.

Table 4: Macro Implications

\begin{tabular}{ccccccc}
\hline \hline Moment & \multicolumn{2}{c}{ Data } & \multicolumn{2}{c}{ Model : Joint } & \multicolumn{2}{c}{ Model : Macro } \\
& estimate & standard error & mean & $90 \%$ interval & mean & $90 \%$ interval \\
\hline$E\left(g_{c, t}\right)$ & 3.23 & 0.2 & 3.36 & {$[3.20,3.56]$} & 3.21 & {$[3.12,3.30]$} \\
$\sigma\left(g_{c, t}\right)$ & 1.84 & 0.16 & 1.88 & {$[1.74,2.02]$} & 1.69 & {$[1.57,1.81]$} \\
$A R_{1}\left(g_{c, t}\right)$ & 0.34 & 0.062 & 0.12 & {$[0.09,0.15]$} & 0.17 & {$[0.12,0.23]$} \\
$A R_{4}\left(g_{c, t}\right)$ & 0.07 & 0.057 & 0.07 & {$[0.05,0.1]$} & 0.10 & {$[0.07,0.14]$} \\
$E\left(\pi_{t}\right)$ & 3.71 & 0.48 & 4.42 & {$[3.69,5.19]$} & 3.71 & {$[3.69,3.75]$} \\
$\sigma\left(\pi_{t}\right)$ & 2.52 & 0.36 & 3.57 & {$[3.49,3.67]$} & 2.30 & {$[2.22,2.39]$} \\
$A R_{1}\left(\pi_{t}\right)$ & 0.84 & 0.048 & 0.62 & {$[0.59,0.64]$} & 0.86 & {$[0.84,0.88]$} \\
$A R_{4}\left(\pi_{t}\right)$ & 0.71 & 0.084 & 0.51 & {$[0.49,0.53]$} & 0.73 & {$[0.71,0.75]$} \\
$\operatorname{Corr}\left(g_{c, t}, \pi_{t}\right)$ & -0.34 & 0.150 & -0.04 & {$[-0.014,-0.071]$} & -0.23 & {$[-0.16,-0.31]$} \\
\hline
\end{tabular}

Notes: Mean and standard deviation of consumption growth and inflation are expressed in terms of annualized percentage. Posterior moments are computed based on 1500 posterior draws obtained by Markov Chain Monte Carlo methods. Standard errors are Newey and West (1987) corrected using 10 lags. 
Table 5: Comparison of Different Estimates of Long Run Consumption RISK

\begin{tabular}{ccc}
\hline \hline & Regression-based Estimates & Filtered Estimates \\
\hline Autocorrelation & 0.634 & 0.888 \\
$R^{2}$ & 0.127 & 0.031 \\
\hline
\end{tabular}

Notes: $R^{2}$ is obtained from the regression of consumption growth on a constant and estimated long run consumption risk.

Table 6: Posterior Mean of Yield Curve Moments

\begin{tabular}{ccccccc}
\hline \hline & $E\left(y_{1, t}\right)$ & $E\left(y_{4, t}\right)$ & $E\left(y_{8, t}\right)$ & $E\left(y_{12, t}\right)$ & $E\left(y_{16, t}\right)$ & $E\left(y_{20, t}\right)$ \\
\hline data & 5.188 & 5.596 & 5.797 & 5.964 & 6.090 & 6.169 \\
model & 5.223 & 5.525 & 5.812 & 5.996 & 6.101 & 6.146 \\
\hline \multirow{2}{*}{ data } & $\sigma\left(y_{1, t}\right)$ & $\sigma\left(y_{4, t}\right)$ & $\sigma\left(y_{8, t}\right)$ & $\sigma\left(y_{12, t}\right)$ & $\sigma\left(y_{16, t}\right)$ & $\sigma\left(y_{20, t}\right)$ \\
model & 2.882 & 2.885 & 2.846 & 2.773 & 2.743 & 2.699 \\
\hline \multirow{2}{*}{ data } & 2.879 & 2.857 & 2.829 & 2.791 & 2.741 & 2.681 \\
model & $0.9 R_{1}\left(y_{1, t}\right)$ & $A R_{1}\left(y_{4, t}\right)$ & $A R_{1}\left(y_{8, t}\right)$ & $A R_{1}\left(y_{12, t}\right)$ & $A R_{1}\left(y_{16, t}\right)$ & $A R_{1}\left(y_{20, t}\right)$ \\
& 0.04 & 0.95 & 0.95 & 0.96 & 0.97 & 0.97 \\
& $E\left(u_{1, t} \mid Y^{T}\right)$ & $E\left(u_{4, t} \mid Y^{T}\right)$ & $E\left(u_{8, t} \mid Y^{T}\right)$ & $E\left(u_{12, t} \mid Y^{T}\right)$ & $E\left(u_{16, t} \mid Y^{T}\right)$ & $E\left(u_{20, t} \mid Y^{T}\right)$ \\
& 0.157 & 0.140 & 0.098 & 0.070 & 0.064 & 0.079 \\
\hline
\end{tabular}

Notes: All the estimates are in terms of the annualized percentage. Posterior moments are computed based on 1500 posterior draws obtained by Markov Chain Monte Carlo methods. The time to maturity is in terms of quarters. $y_{20, t}$ denotes a 5 year bond yield. 
Table 7: Posterior Distribution : P5

\begin{tabular}{cccccc}
\hline \hline Parameter & Prior & \multicolumn{3}{c}{ Posterior } \\
\hline & & \multicolumn{2}{c}{ Joint } & \multicolumn{2}{c}{ Macro } \\
& $90 \%$ Interval & Mean & $90 \%$ Interval & Mean & $90 \%$ Interval \\
\hline$\rho_{11}$ & {$[0.240,0.569]$} & 0.832 & {$[0.812,0.852]$} & 0.345 & {$[0.848,0.889]$} \\
$\rho_{12}$ & {$[-0.167,0.162]$} & 0.003 & {$[-0.002,0.007]$} & -0.123 & {$[-0.197,-0.049]$} \\
$\rho_{21}$ & {$[-0.168,0.160]$} & -0.289 & {$[-0.353,-0.233]$} & 0.132 & {$[0.064,0.201]$} \\
$\rho_{22}$ & {$[0.849,0.994]$} & 0.988 & {$[0.980,0.995]$} & 0.957 & {$[0.925,0.987]$} \\
$\phi_{11}$ & {$[2.050,3.033]$} & 0.246 & {$[0.199,0.295]$} & 2.514 & {$[2.043,2.937]$} \\
$\phi_{12}$ & {$[-0.082,0.081]$} & 0.069 & {$[0.038,0.098]$} & -0.035 & {$[-0.113,0.041]$} \\
$\phi_{21}$ & {$[-0.082,0.082]$} & 0.062 & {$[0.023,0.104]$} & -0.055 & {$[-0.127,0.026]$} \\
$\phi_{22}$ & {$[1.017,1.506]$} & 0.478 & {$[0.428,0.527]$} & 1.139 & {$[0.948,1.339]$} \\
$10000 \sigma_{1}^{2}$ & {$[0.013,0.047]$} & 0.200 & {$[0.177,0.227]$} & 0.025 & {$[0.017,0.033]$} \\
$10000 \sigma_{2}^{2}$ & {$[0.020,0.072]$} & 0.273 & {$[0.240,0.309]$} & 0.035 & {$[0.028,0.042]$} \\
$400 \mu_{1}$ & {$[2.88,3.54]$} & 3.80 & {$[3.63,4.01]$} & 3.23 & {$[3.02,3.44]$} \\
$400 \mu_{2}$ & {$[3.27,3.93]$} & 3.54 & {$[3.21,3.84]$} & 3.58 & {$[3.25,3.91]$} \\
$\delta$ & {$[0.9942,0.9998]$} & 0.997 & {$[0.9953,0.9989]$} & 0.997 & fixed \\
$\psi$ & {$[1.164,1.818]$} & 1.775 & {$[1.517,2.081]$} & 1.5 & fixed \\
$\gamma$ & {$[12.174,21.927]$} & 9.810 & {$[7.327,11.899]$} & 17.000 & fixed \\
\hline
\end{tabular}


Figure 1: Inflation and Long Term Interest Rate

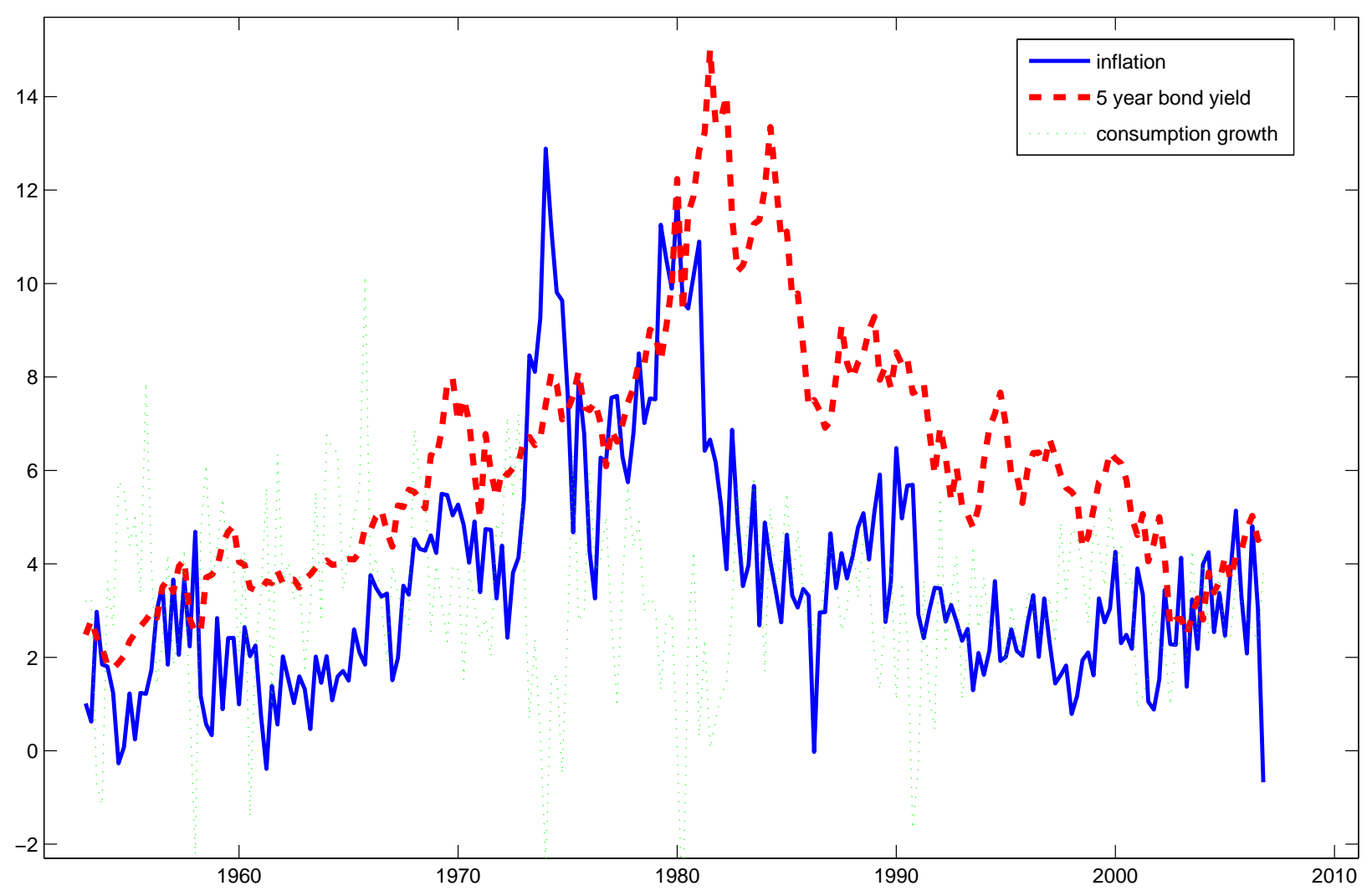


Figure 2: Realized and Expected Consumption Growth

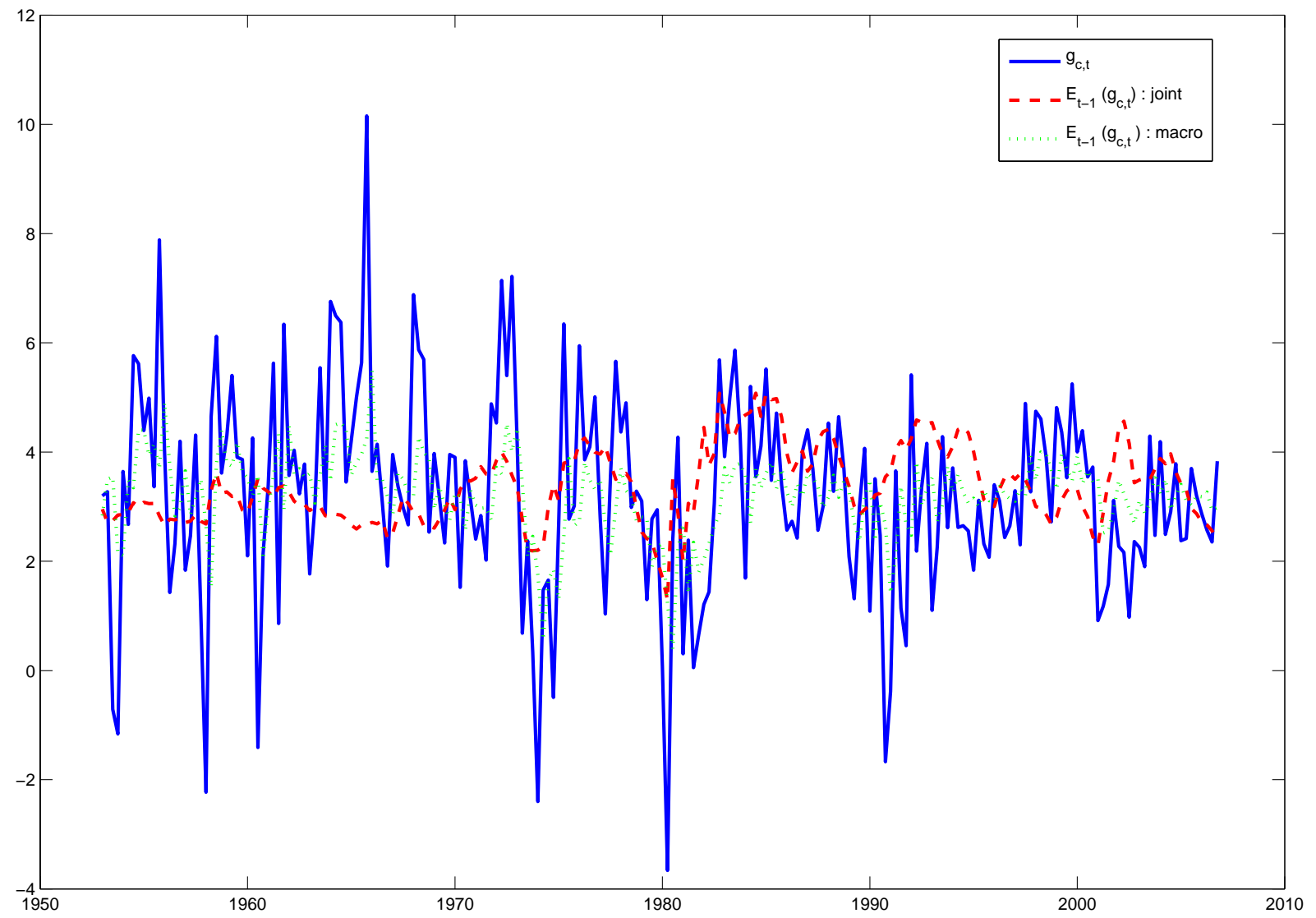

Expected consumption growth is obtained by running Kalman smoothing at the posterior mean of parameters. The joint estimation uses term structure data as well as macro data. 
Figure 3: REALIZED AND EXPECTED INFLATION

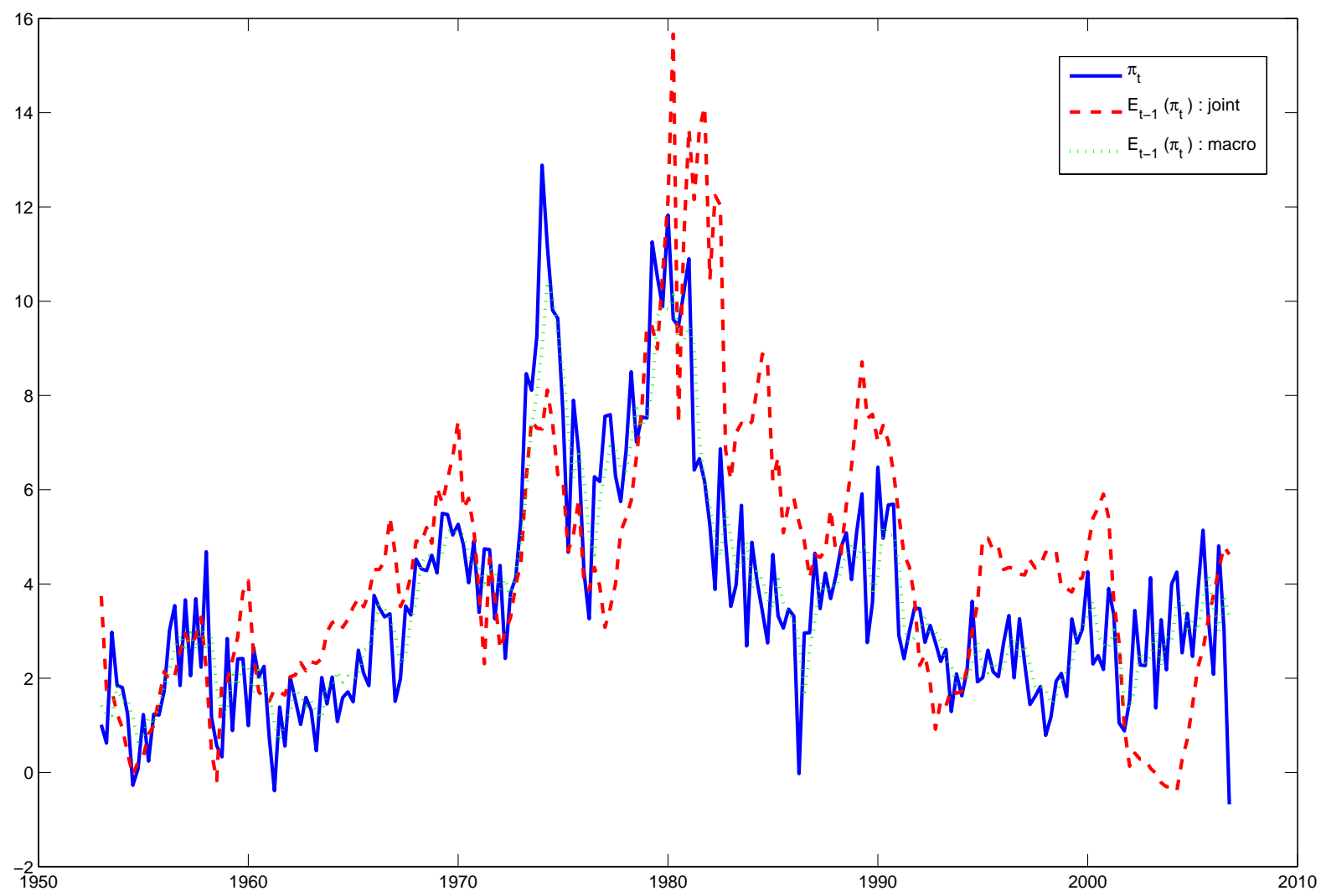

Expected inflation is obtained by running Kalman smoothing at the posterior mean of parameters. The joint estimation uses term structure data as well as macro data. 
Figure 4: Comparison of Estimates of Expected Consumption Growth

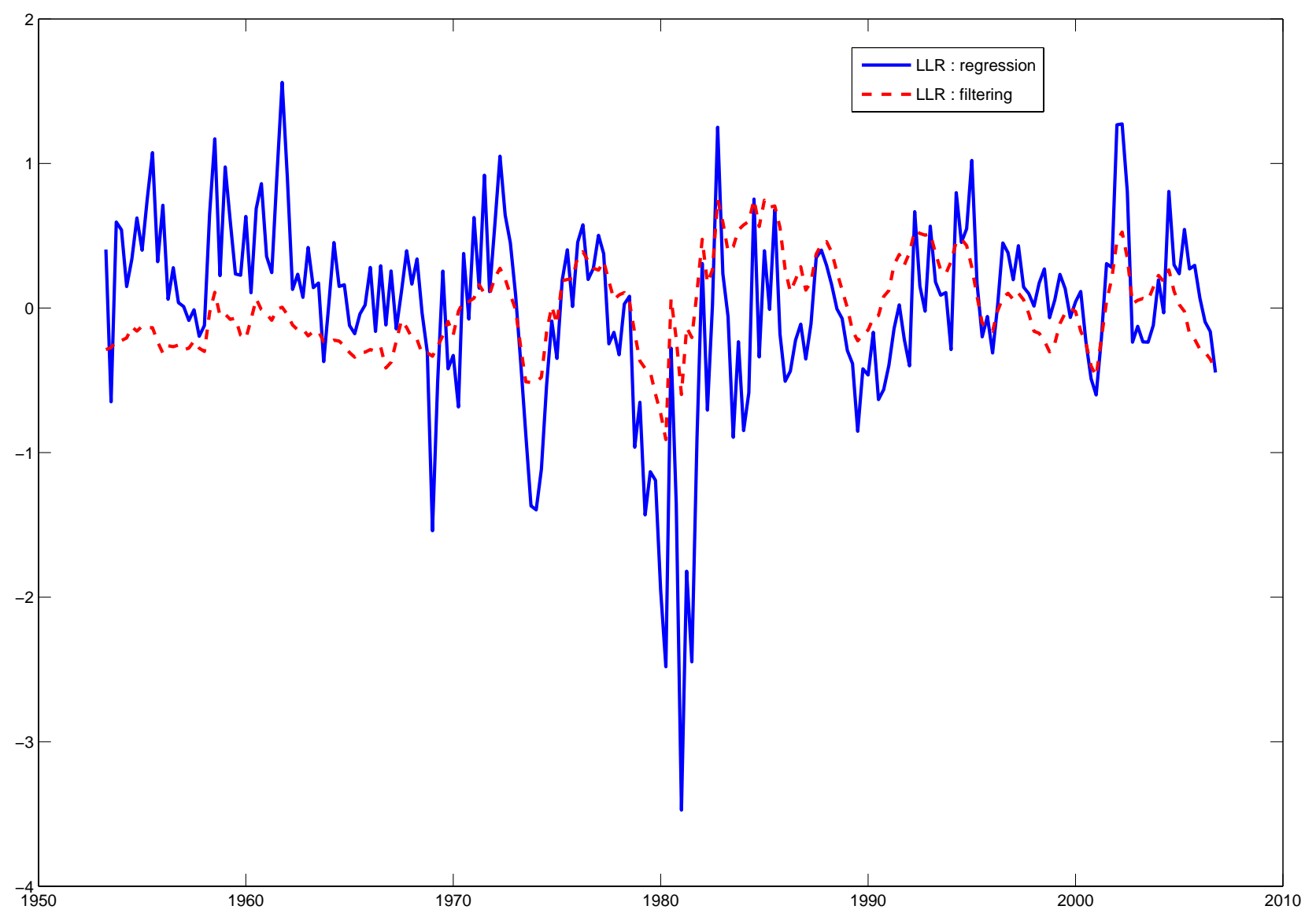

The solid line corresponds to the expected consumption growth obtained from regressing observed consumption growth data on term structure variables. The dash line denotes the estimates of expected consumption growth obtained by running Kalman smoothing at the posterior mean of parameters. 